\title{
Research on Digital Remote Control Technology of Surveying and Unmanned Aerial Vehicle
}

\author{
Na Zhang ${ }^{1, a}$ \\ ${ }^{1}$ Liaoning Jianzhu Vocational College, Liaoyang, Liaoning, 111000 \\ ${ }^{a}$ email
}

Keywords: Remote Control, Surveying and Unmanned Aircraft, Control Technology

\begin{abstract}
UAV remote control driving mode relative to the program-controlled way, in the time delay, scenarios, data link performance optimization and flight control and other aspects of the higher requirements, and these factors have become troubled UAV remote control driving development and application the essential. Based on the results of UAV technology to verify the ground closed-loop test of the unmanned aerial vehicle (UAV) technology, the key technical difficulties faced by the remote control driving of UAV are analyzed with the emphasis on time delay and data link performance. Technical solution and the use of ground closed-loop test environment to verify and finally the use of manned flight quality standards proposed a preliminary UAV flight quality evaluation method.
\end{abstract}

\section{Introduction}

Remote sensing flight platform is divided into satellite remote sensing and aeronautical remote sensing, aerial remote sensing platform in the past is mainly someone aircraft. In the 1990s, with the rapid development of electronic technology, small unmanned aerial vehicles in the remote control, life time, flight quality has been a clear breakthrough, became the recent rise of the new remote sensing means, and in the remote sensing sector is generally considered to have good prospects for development. Compared with the man-machine, the advantages of unmanned aerial vehicles are mainly manifested in a very high mobility, all the equipment together is 100 to kilograms, in the motor speed, maneuver range, motor conditions, etc., is any the aircraft cannot be compared with the second is a strong environmental adaptability, no special landing space, where to fly where the meteorological conditions are very low requirements, superior low-level performance makes the cloud operation becomes easy, which greatly improve the efficiency of the three is an excellent economy, the aircraft to buy cheap, the average company can afford, the use of low cost, and do not need manned, the user's safety pressure greatly reduced. From the performance of the aircraft, UAV and man-machine is an important difference is that the unmanned aerial vehicle in the horizon of the flight, completely by the autopilot by the default procedure cannot be carried out according to the actual flight situation is no human intervention small volume can be loaded space and weight is very limited can only load a small ordinary sensor. Third, the UAV flight by the airflow disturbance caused by the flight state deviation, mainly by the aircraft's own flight stability to restore, so there is Obviously slow. The above characteristics directly affect the quality of aerial photography, with UAV aerial photography, often the image quality is not high, overlap error, missed and so on.

\section{The Mapping Requirements of UAV Technology}

Surveying and mapping industry on the aerial photography requirements are relatively high and photogrammetry requirements of the camera image strict distortion correction, in flight must be required to strictly ensure that the course and the direction of overlapping cannot be less than the side cannot be less than the dagger in the process of rotation. The declination must be small enough that the skew angle should be controlled within the chance and so on. Therefore, the system to adapt to the mapping for a photogrammetric post-processing requirements, corresponding to the following three aspects of improvement in the aerial photography before the study of the camera's precision calibration and image distortion correction, developed a spin correction cloud In order to correct the 
flying angle of the spine, the designated exposure device was designed to control the exposure of the camera in the set exposure range to ensure that the heading and the side overlap.

Digital camera calibration includes the determination of the measurement of the main distance, the determination of the optical distortion coefficient. The error of the digital camera is composed of optical error, mechanical error and electrical error. Optical error mainly refers to the optical distortion error, that is, the camera body lens system, assembly caused by the image point deviates from its ideal position of the bit error, it is divided into radial distortion and eccentric distortion difference between the two types of electrical errors include line synchronization error, field synchronization error and sampling error mechanical error refers to the error from the image taken from the optical lens to the digital array image.

The indoor control field is composed of some known high-precision spatial coordinates of the sign point. Indoor control field is generally uniform distribution of a certain number of two-dimensional or two-dimensional control signs can carry light metal frame, indoor control field itself should be strong, no deformation, easy to carry and so on, for the convenience of use, you can use light metal pipe Made a shape of the framework, and in the appropriate part of the framework of the layout of some control points, control the coordinates of the field points through the high-precision theodolite to the intersection of the way to determine.

There are many ways to check the camera, commonly used computer vision methods and photogrammetric methods two categories. The basic principle of the two-step method in computer vision is to acquire the image with the camera with the coordinates of the space point, and use the sub-pixel image localization technique to extract the coordinates of the image grid point, and use the corresponding relationship between the different image grid points camera calibration. This method is not calculated, the accuracy is moderate, is the computer vision commonly used in the calibration method. However, the main points of this method need to be pre-calibrated by other methods, and only consider the radial distortion, when the tangential distortion is not applicable.

\section{Remote Control Driving Key Technical Analysis}

In the UAV system, the meaning of the time delay refers to the time from the ground driver's input to the time he felt the experience of the unmanned aerial vehicle response. According to the composition of UAV system, UAV system time delay mainly includes the signal acquisition and processing, data transmission and display, platform response generated by the time delay. The time delay caused by the general signal acquisition and processing is related to the running period of the system software. Normally, the running time of the system software is generally controlled between 10 and $20 \mathrm{~ms}$. Therefore, the time delay caused by the running of the system software is about 60 ms System software running cycle to take the median value of $15 \mathrm{~ms}$ ). In contrast, the data link upstream and downstream transmission and platform response time is longer, in the UAV system time delay in the total occupies the main position, usually without the use of satellite relay case, the data link up and down transmission time to maintain Between 110 and 120 ms, the UAV platform response time is usually around $100 \mathrm{~ms}$, and it appears that the total amount of time delay of the UAV system will normally be around $280 \mathrm{~ms}$.

The data link is responsible for the transmission of data on the upstream and downstream data, which has a vital role in the performance, reliability and safety of the whole UAV system. The performance of the data link is good and the management strategy is directly affected. The main indicators of data link performance include transmission delay, frame loss rate and bit error rate. As the data link transmission delay in the whole system of the total time delay in the proportion of large, large time delay will cause the system real-time decline, and for the UAV flight quality will have a greater impact. Framing rate has a certain impact on the system delay, under normal circumstances will not dominate, but if the duration of the lost frame for the system time delay will have a more significant impact. 


\section{Remote Control Driving Key Technology Solutions}

Time delay has great influence on flight quality. At present, most of the time delay of UAV system is about $400 \mathrm{~ms}$, and the flight quality is very poor in remote driving mode. It can only complete some simple tasks and cannot achieve rapid maneuvering. In order to improve the flight quality of UAV remote control driving, a large number of research and experiment verification work has been carried out in this field, and a time delay compensation technology with practical application value has been put forward. The results of the foreign operator evaluation show that the operator will give Grade 3 flight quality and $\mathrm{CH}$ rating of 7 to 9 (also known as Cooper-Harper Flight Quality Assessment) when the system time is delayed by 189 ms without compensation technology Level, created by Cooper-Harper, divided into 10 levels), and the use of time delay compensation technology, the system time delay of $389 \mathrm{~ms}$, the operator can give two flight quality and 5 . 3 level $\mathrm{CH}$ Rating, which means that the use of time delay compensation technology to improve the flight quality is feasible, and very effective. The basic principle of time delay compensation technology is to use the aircraft response prediction and correction technology to achieve the system time delay compensation to achieve the time delay to reduce the "illusion" to improve the driver when the flight quality. The realization of the aircraft through the ground station in the establishment of the dynamics of the aircraft model, when the pilot to manipulate, the first through the dynamic model to predict the response to the aircraft, the forecast response to the operator first, and then with the link down The real flight parameters are superimposed and corrected to ensure consistency between the predictive response and the actual response, thereby improving the operator \& apos; s operational experience.

Some of the characteristics of the data link for the flight quality and even flight safety has a crucial impact, in order to improve the UAV flight quality characteristics, to eliminate the characteristics of the data chain itself caused by security risks, the need for effective and reliable data chain management strategy to optimize its performance to ensure system security, improve flight quality. In the actual application process, due to the data link on the downlink transmission mechanism is limited, the data link time delay and frame loss rate will have some interaction, real-time increase will lead to data link frame loss rate increases, the rate will also result in an increase in the data link transmission time delay, which may reach a maximum of $1 \mathrm{~s}$ or more. As the uplink and downlink data transmission airborne flight control or ground data acquisition between the computer and the measurement and control links will exist between the time is not synchronized, so the airborne flight control and ground data acquisition computer from the monitoring link to receive the data may not be Is a complete data frame. To reduce the frame loss rate, you need airborne flight control, the ground data acquisition computer on the received data for the second frame combination, which may lead to data accumulation, resulting in increased system time delay. As the remote control mode up and down the amount of information transmission, will occur frequently data accumulation, which will seriously affect the real-time data transmission. If you want to improve real-time, you must "abandon" the uplink and downlink transmission of data in the incomplete frame, which will lead to a substantial increase in frame loss rate, which requires different modes of operation to choose a different approach. Program mode requires a lower data transmission frame rate, improve the continuity of data transmission, remote control driving mode has a high real-time requirements, this time under the conditions allowed to improve the frame rate to meet the right Real-time requirements. Through a large number of ground tests show that in the case of real-time control of the required remote control mode, the transmission frame loss rate of about $16 \%$, while the test also shows that a reasonable approach to ensure that $16 \%$ of the frame rate of aircraft Remote control driving operation will not have a significant impact.

Under normal conditions of use, the duration of frame dropping is about 30 to $45 \mathrm{~ms}$ and does not have a significant effect on the aircraft platform. If there is electromagnetic interference or occlusion, there may be more than $1 \mathrm{~s}$ continuous frame dropping, then for remote control driving is unbearable. In order to ensure system security, according to the time of continuous frame loss to take different emergency measures, such as smooth transition and mode switching. The processing 
of the bit error rate requires the speed and amplitude to be limited when the remote control driving command is received to ensure the continuity and correctness of the steering instruction. In order to avoid the large transient response of the aircraft platform due to the occurrence of the error, Of the safety, need to set the appropriate safety emergency measures.

\section{Conclusion}

Measurement and control of data link performance and time delay is the impact of UAV remote control driving quality characteristics of the two very important factors, if there is no reasonable management strategy and compensation methods, will affect the UAV quality characteristics and even security. The ground test proved that this method can effectively improve the flight quality characteristics and safety of UAV system and it is suitable for engineering application. The establishment of UAV flight quality is based on a large number of test data, and for different forms of operation and task objectives to refine the requirements for remote control driving flight quality requirements in the time delay and data link performance requirements should be considered.

\section{References}

[1] Zhang Jianqing, Ke Tao, Sun Mingwei, Duan Yansong.Application and Implementation of Parallel Computing in Aerial Photogrammetry[J]. Bulletin of Surveying and Mapping. 2008 (12)

[2] Yang Guoliang. Design and implementation of small unmanned aerial vehicle ground control station software [J]. Telemetry and Remote Control. 2008 (03)

[3] Li Bing, Yue Jingxian, Li Hejun. UAV photogrammetry technology exploration and application research [J]. Beijing Surveying and Mapping. 2008 (01)

[4] Han Jie, Wang Zheng. Unmanned aerial vehicle remote sensing land resources rapid monitoring system key technology research [J]. Bulletin of Surveying and Mapping. 2008 (02)

[5] Cui Hongxia. Fabrication of Large Scale Orthophoto Images Based on Unmanned Aerial Vehicle System [J]. Electronic Devices. 2008 (01)

[6] Wu Yudong, Zhang Qiang. No-helicopter low-altitude digital photography and image measurement technology[J] .Journal of Surveying and Mapping. 2007 (05)

[7] Zhang Zuxun.From Digital Photogrammetry Workstation (DPW) to Digital Photogrammetric Grid (DPGrid)[J]. Journal of Wuhan University. 2007 (07) 